\title{
An Explicit Criterion for Existence of the Magnus Solution for a Coupled Spin System under a Time-Dependent Radiofrequency Pulse日
}

\author{
Xijia Miao \\ Laboratory of Magnetic Resonance and Atomic and Molecular \\ Physics, Wuhan Institute of Physics and Mathematics, The \\ Chinese Academy of Sciences, Wuhan 430071, P. R. China \\ (Current Email: miaoxijia@yahoo.com)
}

\begin{abstract}
The explicit criterion is derived in detail for the convergence of the Magnus expansion and the existence of the Magnus solution in the interaction picture, i.e., the exponential propagator in the weakly coupled spin $(\mathrm{I}=1 / 2)$ system $\mathrm{S}_{n}$ AMX... $(\mathrm{n}=1,2,3, \ldots)$ in which only spin group $S$ is subjected to a timedependent shaped selective radiofrequency pulse. The derivation is built on the same scheme as the Maricq's (J. Chem. Phys. 86 (1987) 5647). It is shown that the criterion depends only upon amplitude of the time-dependent field applied to the system, and the Magnus expansion converges and the Magnus solution exists when the flip angle of a non-negative-amplitude shaped RF pulse or a weak-amplitude shaped pulse is smaller than $2 \pi$. The exponential propagator then can be decomposed into a product of a series of elementary propagators and can be used to determine time evolution of the spin system under the shaped pulse. The linear differential equations to determine the unknown parameters in the Magnus solution are obtained explicitly. An alternative propagator in an expansion form for the Magnus solution is also proposed to describe the time evolution when the criterion is not met.
\end{abstract}

PACS: 03.65.Ge; 33.25.+k2

Keywords: Convergent criterion of the Magnus expansion; Exponential solution to the Schrödinger equation; Time-dependent radiofrequency field; The shaped selective RF pulse; Nuclear magnetic resonance.

The Magnus expansion [1] plays an important role in determination for time evolution of quantum systems under time-dependent Hamiltonian. The advantage of the Magnus expansion over the standard quantum mechanical perturbation theory is that Hermiticity is retained to each order in the expansion. The

\footnotetext{
${ }^{1}$ This paper is the corrected version of the original manuscript (the year 2000) and its published paper (Xijia Miao, Phys. Lett. A 271 (2000) 296-302). There was not proof of the published paper. All the error corrections, modifications, and comments are noted clearly in the footnotes in this corrected paper. The corrected paper, the original manuscript and/or the published paper appear in the website Arxiv.org with the same paper ID and different version.

${ }^{2}$ This row of the original manuscript is already corrected here.
} 
Magnus expansion has an extensive application in nuclear magnetic resonance (NMR) spectroscopy: the average Hamiltonian theory [2, 3] which is based on the Magnus expansion has been used extensively in the design and analysis of a variety of multipulse NMR experiments both in liquid and in solid states. The Magnus expansion has also been used successfully to investigate the response of coupled spin systems to a time-dependent radiofrequency (RF) field, e.g., a shaped selective pulse $[4,5]$. The Magnus solution, namely, the continuous exponential solution to the time-dependent Schrödinger equation, has recently been used to characterize the behavior of a coupled spin system under the timedependent radiation damping field [6]. Although the Magnus expansion and the Magnus solution are applied conveniently to a variety of time-dependent systems, there are strict limits on the existence of the solution and there is also a possible serious problem for the expansion concerning its convergence $[1,7$ 9]. As pointed out by Magnus [1] as long ago as in 1954, the Magnus solution $U(t)=\exp [-i \Omega(t)]$ exists only when the so-called Magnus criterion is satisfied: $\left|\lambda_{i}(t)-\lambda_{j}(t)\right| \neq n 2 \pi$, where $\lambda_{i}(t)$ are the eigenvalues of the operator $\Omega(t)$ and $n$ is a nonzero integer. This criterion is an implicit one and has little value since $\lambda_{i}(t)$ are the unknown quantity under investigation. An explicit criterion $3^{3}$ is therefore needed for the convenient application of the Magnus solution. Maricq [8] showed that the operator $\Omega(t)$ exists for short evolutional times and the Magnus expansion always converges in the neighborhood of the time $t=0$. Maricq [9] further found that the convergence for an isolated two-level system under a linear polarized field can be improved: the Magnus expansion is convergent and hence the Magnus solution exists in time interval $[0, \tau]$ if the following inequality is satisfied

$$
\int_{0}^{\tau}\left|\omega\left(t^{\prime}\right)\right| d t^{\prime}<2 \pi
$$

where $\pm \omega(t) / 2$ are the two eigenvalues of the time-dependent Hamiltonian $H(t)$ of the system. The advantage for this criterion is that it is dependent only on the eigenvalues of the Hamiltonian $H(t)$. However, an explicit criterion may be far from the simple form of inequality (1) and has not yet been found for a general time-dependent multilevel system [7 - 9].

A shaped selective pulse is an amplitude-modulation and/or phase-modulation RF field [4, 10 - 13]. The Magnus solution may provide a convenient starting point for an exact description of time evolution of coupled spin systems under a time-dependent shaped selective RF pulse. However, so far an explicit criterion 4 for existence of the Magnus solution and convergence of the Magnus expansion has not been 5 established for the general spin systems. This general problem could not be settled simply, but in some special cases it may be able to be solved without much difficulty. In this communication the Maricq's criterion (1) for a single two-level system is extended to the weakly coupled spin $(I=1 / 2)$ systems

\footnotetext{
${ }^{3}$ The original words are modified here. The original word(s) appears in the original manuscript and its published paper (Xijia Miao, Phys. Lett. A 271 (2000) 296-302). Hereafter use italics to mark the modified word(s).

${ }^{4}$ The original word is modified here.

${ }^{5}$ The original word is modified here.
} 
$\mathrm{S}_{n}$ AMX .. $\quad(\mathrm{n}=1,2, \ldots)$, where only spin $\mathrm{S}$ is undergone a time-dependent $\mathrm{RF}$ field. It is known that the properties of the Magnus expansion depend strongly on the representation [7 - 9]. The present criterion is derived explicitly in the interaction picture defined by spin Hamiltonian of the spin system in the absence of the time-dependent RF field. In this representation the criterion is dependent only upon the amplitude of the time-dependent RF field applied to the system. Therefore, in practice this is quite convenient to use the criterion.

For simplification, consider the SAMX... weakly coupled spin $(\mathrm{I}=1 / 2)$ system in which only spin $\mathrm{S}$ is subjected to a time-dependent RF field, e.g., a shaped selective pulse. The contribution of the time-dependent RF field to spin Hamiltonian of the system is given by

$$
H_{1}(t)=\omega_{1}(t)\left\{S_{x} \cos \phi(t)+S_{y} \sin \phi(t)\right\}
$$

where $\omega_{1}(t)$ and $\phi(t)$ are known as the amplitude and phase of the timedependent RF field, respectively. Then the total spin Hamiltonian of the spin system in a strong static magnetic field can be written in the rotating frame

$$
H(t)=H_{0}+H_{1}(t)
$$

where $H_{0}=H_{I}+\Omega_{I} S_{z} ; H_{I}=\sum_{k} \Omega_{k} I_{k z}+\sum_{k<l} \pi J_{k l} 2 I_{k z} I_{l z}$ and $\Omega_{I}=\Omega_{s}+$ $2 \sum_{k} \pi J_{k s} I_{k z}$; the symbol $I$ denotes the spin A, M, X, .... Obviously, both the 6 operators $H_{I}$ and $\Omega_{I}$ are members of the longitudinal magnetization and $s$ pin order $(L O M S O)$ operator subspace of the Liouville operator space of the spin subsystem AMX... of the system SAMX... [14]. They 7 commute with each other because any two base operators of the LOMSO operator subspace commute with each other [14]. They also commute with the magnetization operators $\left\{S_{x}, S_{y}, S_{z}\right\}$ of spin $S$. The time-evolutional propagator corresponding to the time-dependent Hamiltonian (3) can be written as

$$
U(t)=T \exp \left(-i \int_{0}^{t} H\left(t^{\prime}\right) d t^{\prime}\right)=\exp \left(-i H_{0} t\right) U_{I}(t)
$$

where $T$ is Dyson time-ordering operator and the propagator $U_{I}(t)$ in the interaction representation is given by

$$
U_{I}(t)=T \exp \left(-i \int_{0}^{t} H_{i}\left(t^{\prime}\right) d t^{\prime}\right)
$$

with the time-dependent Hamiltonian in the interaction representation defined by the unperturbed Hamiltonian $H_{0}$ of Eq. (3):

$$
H_{i}(t)=\exp \left(i H_{0} t\right) H_{1}(t) \exp \left(-i H_{0} t\right)
$$

By expanding the right-hand side of equation (6) according to the rotation transformations [2] between any two product operators and the fact that $\left[H_{I}\right.$, $\left.\Omega_{I}\right]=0$, the Hamiltonian $H_{i}(t)$ is reduced to an explicit expression

$$
H_{i}(t)=\omega_{1}(t) \boldsymbol{h}(\boldsymbol{t}) . \boldsymbol{S}=\omega_{1}(t)\left\{h_{x}(t) S_{x}+h_{y}(t) S_{y}+h_{z}(t) S_{z}\right\}
$$

\footnotetext{
${ }^{6}$ Here drops a redundant original word.

${ }^{7}$ Here drops a redundant original word.
} 
where the operator $h_{x}(t)=\cos \left(-\Omega_{I} t+\phi(t)\right), h_{y}(t)=\sin \left(-\Omega_{I} t+\phi(t)\right)$, and $h_{z}(t)=0$. Because $h_{x}(t)^{2}+h_{y}(t)^{2}+h_{z}(t)^{2}=E$ (unit operator), the operator $\boldsymbol{h}(\boldsymbol{t})=\left(h_{x}(t), h_{y}(t), h_{z}(t)\right)$ is an operator vector with the unit magnitude. Obviously, the operator $\boldsymbol{h}(\boldsymbol{t})$ belongs the LOMSO operator subspace of the subsystem AMX.... It follows from Eq. (7) that

$$
\left\|H_{i}(t)\right\| \leq\left|\omega_{1}(t)\right| \cdot|| \boldsymbol{h}(\boldsymbol{t})\|\cdot\| \boldsymbol{S} \|
$$

Therefore, all eigenvalues of the Hamiltonian $H_{i}(t)$ are bounded by the amplitude $\left|\omega_{1}(t)\right|$ in magnitude of the time-dependent RF field. In a single-spin system $\boldsymbol{h}(\boldsymbol{t})$ is a vector of simple scalars and in this case the criterion, that is, inequality (1), was obtained explicitly by Maricq [9], but in the SAMX... coupled spin system $\boldsymbol{h}(\boldsymbol{t})$ can be taken as a vector of the operators $h_{p}(t)(p=x, y, z)$ that all commutes one with each other because the operators $h_{p}(t)$ are members of the LOMSO operator subspace. In the present more general case the criterion of the Magnus expansion in the interaction picture has not been obtained explicitly and it is just the purpose of the present work to extend the criterion (1) to the $\mathrm{S}_{n} \mathrm{AMX}$... coupled spin systems.

In order to determine exactly and analytically time evolution of the coupled spin system under a time-dependent RF field one needs to solve the timedependent Schrödinger equation or equivalent Liouville-von Neumann equation. However, it is usually difficult to solve exactly the time-dependent dynamical equations and therefore to determine exactly the density operator and the response of the system to the time-dependent RF field. A numerical calculation is a general routine to solve time-dependent problems, but it is usually not much helpful for one to gain a deeper insight into the physical essence for the effect of a time-dependent RF field on the time evolution and for the effect of scalar coupling on the response. The approximated treatments that usually employ the perturbation theory or the average Hamiltonian theory are valuable, but there are some limitations and disadvantages of these approximated methods and therefore, an exact solution to the time-dependent problems is still attractive. One strategy to determine exactly and analytically time evolution of the coupled spin system under a time-dependent RF field is that the propagator $U_{I}(t)$ of Eq. (5) is written as an exponential operator without the Dyson timeordering operator $\mathrm{T}$, as suggested by Magnus in early day [1]. Note that the magnetization operators $\left\{E, S_{x}, S_{y}, S_{z}\right\}$ of the spin $S$ form the $u(2)$ Lie algebra subspace and the longitudinal magnetization and spin order operators $\left\{E, I_{k z}\right.$, $\left.2 I_{k z} I_{l z}, 4 I_{k z} I_{l z} I_{m z}, \ldots\right\}$ of the subsystem AMX... form the LOMSO operator subspace [14] by including the unit operator $E$. Then the complete operator set $\left\{E, S_{p}, I_{k z}, 2 S_{p} I_{k z}, 2 I_{k z} I_{l z}, 4 S_{p} I_{k z} I_{l z}, \ldots ; p=x, y, z\right\}$ is a closed operator algebra subspace of the Liouville operator space of the spin system SAMX... and actually, this subspace is the direct product space of the subspaces $u(2)$ and $L O M S O$, that is, $G=u(2) \bigotimes L O M S O$ [14]. Obviously, the Hamiltonian $H_{i}(t)$ of Eq. (7) is a member of the operator subspace $G$ because the operators $h_{p}(t)$ $(p=x, y, z)$ in Eq. (7) are members of the LOMSO operator subspace. Then the propagator $U_{I}(t)$ of Eq. (5) corresponding to the time-dependent Hamilto- 
nian $H_{i}(t)$ is also a member of the subspace $G$ [14] and may be conveniently written as an exponential unitary operator:

$$
U_{I}(t)=\exp [-i \Omega(t)]
$$

with the Hermitian operator

$$
\Omega(t)=\Omega_{x}(t) S_{x}+\Omega_{y}(t) S_{y}+\Omega_{z}(t) S_{z}
$$

where the Hermitian operator $\Omega(t)$ is an element of the operator subspace $G$ and $\Omega_{p}(t)(p=x, y, z)$ belong the LOMSO operator subspace. The exponential propagator of Eq. (9) is quite simple and can be further decomposed into a sequence of elementary propagators $[15,16]$

$$
\begin{aligned}
& U_{I}(t)=\exp (\left.-i \alpha(t) S_{z}\right) \exp \left(-i \beta(t) S_{y}\right) \exp \left(-i \hat{\Omega}(t) S_{z}\right) \\
& \times \exp \left(i \beta(t) S_{y}\right) \exp \left(i \alpha(t) S_{z}\right)
\end{aligned}
$$

where the operators $\alpha(t), \beta(t)$, and $\hat{\Omega}(t)$ that are also members of the LOMSO operator subspace are related to the operators $\Omega_{x}(t), \Omega_{y}(t)$, and $\Omega_{z}(t)$ in Eq. (10) by the following formulae:

$$
\begin{gathered}
\Omega_{x}(t) \sin \alpha(t)=\Omega_{y}(t) \cos \alpha(t) \\
\Omega_{z}(t) \sin \beta(t)=\left[\Omega_{x}(t) \cos \alpha(t)+\Omega_{y}(t) \sin \alpha(t)\right] \cos \beta(t) \\
\hat{\Omega}(t)=\Omega_{z}(t) \cos \beta(t)+\left[\Omega_{x}(t) \cos \alpha(t)+\Omega_{y}(t) \sin \alpha(t)\right] \sin \beta(t)
\end{gathered}
$$

The exponential propagator of Eq. (9) of the SAMX... spin system can be extended to the coupled spin systems $\mathrm{S}_{n}$ AMX $\ldots(\mathrm{n}=1,2,3, \ldots)$ and in this more general case the propagator can be written a 8

$$
U_{I}(t)=\prod_{k=1}^{n} \exp \left[-i\left(\Omega_{x}(t) S_{k x}+\Omega_{y}(t) S_{k y}+\Omega_{z}(t) S_{k z}\right)\right]
$$

With the help of the decomposed propagator of Eq. (11) one can determine conveniently time evolution of the coupled spin system SAMX... under a shaped pulse. However, there are two problems that need to be solved in advance. One of which is that the existence of the exponential propagator $U_{I}(t)$ of Eq. (9) needs to be verified. This would be achieved by setting up the convergence condition of the Magnus expansion of the operator $\Omega(t)$ in Eq. (9) and hence

\footnotetext{
is

${ }^{8}$ The incorrect expression (13) appearing in the original manuscript and its published paper$$
U_{I}(t)=\prod_{k=1}^{n} \exp \left[-i \Omega_{x}(t) S_{k x}+\Omega_{y}(t) S_{k y}+\Omega_{z}(t) S_{k z}\right]
$$ 
the existence condition of the Magnus solution of Eq. (9) in the interaction picture. Another is that the unknown parameters in the decomposed propagator of Eq. (11) should be determined explicitly. It was shown by Maricq [9] that the explicit condition (1) for the convergence of the Magnus expansion in a singlespin system under a linear polarized field is related only to the eigenvalues of the time-dependent Hamiltonian of the system. However, it has not been clear whether the convergence of the Magnus expansion in the coupled spin system 9 SAMX... is still dependent only upon the eigenvalues of the time-dependent Hamiltonian of the system. From the point of view of convenient application, the convergence had better depend only on the eigenvalues of the Hamiltonian or even only upon the amplitude and/or phase of the time-dependent RF field applied to the system in the interaction frame. On the other hand, it is expected that the convergence of the Magnus expansion in the interaction frame may depend only upon the amplitude of the time-dependent RF field as all the eigenvalues of the time-dependent Hamiltonian (6) in the interaction frame are bounded on by the amplitude of the RF field in magnitude (see inequality (8)). Therefore, in the following the convergence criterion is derived in the interaction representation enabling it to depend only upon the amplitude of the RF field. The derivation is based on the decomposed propagator of Eq. (11) and the time-dependent Schrödinger equation in the interaction frame:

$$
\frac{d}{d t} U_{I}(t)=-i H_{i}(t) U_{I}(t) \quad(\hbar=1)
$$

The decomposed propagator of Eq. (11) is first expanded as a linear combination of the magnetization operators $S_{p}(p=x, y, z)$

$$
\begin{gathered}
U_{I}(t)=\cos \left(\frac{1}{2} \hat{\Omega}(t)\right)-2 i\left[S_{z} \cos \beta(t)+S_{x} \cos \alpha(t) \sin \beta(t)\right. \\
\left.+S_{y} \sin \alpha(t) \sin \beta(t)\right] \sin \left(\frac{1}{2} \hat{\Omega}(t)\right)
\end{gathered}
$$

Equation (15) is the expansion expression of the propagator $U_{I}(t)$ in the operator subspace $G$. Then inserting both the Hamiltonian $H_{i}(t)$ of Eq. (7) and the expansion-form propagator $U_{I}(t)$ of Eq. (15) into equation (14) one obtains a set of equations entirely equivalent to the time-dependent Schrödinger equation (14)

$$
\begin{gathered}
\frac{d}{d t} \cos \left(\frac{1}{2} \hat{\Omega}(t)\right)=-\frac{1}{2} \omega_{1}(t) \sin \left(\frac{1}{2} \hat{\Omega}(t)\right)\left\{h_{x}(t) \cos \alpha(t) \sin \beta(t)\right. \\
\left.+h_{y}(t) \sin \alpha(t) \sin \beta(t)+h_{z}(t) \cos \beta(t)\right\} \\
\frac{d}{d t}\left[\cos \alpha(t) \sin \beta(t) \sin \left(\frac{1}{2} \hat{\Omega}(t)\right), \quad \sin \alpha(t) \sin \beta(t) \sin \left(\frac{1}{2} \hat{\Omega}(t)\right), \quad \cos \beta(t) \sin \left(\frac{1}{2} \hat{\Omega}(t)\right)\right] \\
=\frac{1}{2} \omega_{1}(t) \cos \left(\frac{1}{2} \hat{\Omega}(t)\right)\left[h_{x}(t), h_{y}(t), h_{z}(t)\right]
\end{gathered}
$$

${ }^{9}$ The original word is modified here. 


$$
\begin{gathered}
+\frac{1}{2} \omega_{1}(t) \sin \left(\frac{1}{2} \hat{\Omega}(t)\right)\left[-h_{z}(t) \sin \alpha(t) \sin \beta(t)+h_{y}(t) \cos \beta(t),\right. \\
h_{z}(t) \cos \alpha(t) \sin \beta(t)-h_{x}(t) \cos \beta(t), \\
\left.h_{x}(t) \sin \alpha(t) \sin \beta(t)-h_{y}(t) \cos \alpha(t) \sin \beta(t)\right]
\end{gathered}
$$

Equations (16) 10 are a set of linear coupled differential equations with the operator variables: $\cos \left(\frac{1}{2} \hat{\Omega}(t)\right), \cos \alpha(t) \sin \beta(t) \sin \left(\frac{1}{2} \hat{\Omega}(t)\right), \sin \alpha(t) \sin \beta(t) \sin \left(\frac{1}{2} \hat{\Omega}(t)\right), 11$ and $\cos \beta(t) \sin \left(\frac{1}{2} \hat{\Omega}(t)\right)$ of the LOMSO operator subspace. They can be used to determine generally the unknown parameters in the decomposed propagator of Eq. (11). Also, from them one may extract the criterion of convergence of the Magnus expansion in the interaction frame. Because any two base operators of the LOMSO subspace commute and the operator $\hat{\Omega}(t)$ belongs the LOMSO subspace one has

$$
\frac{d}{d t} \cos \left(\frac{1}{2} \hat{\Omega}(t)\right)=-\frac{1}{2} \sin \left(\frac{1}{2} \hat{\Omega}(t)\right) \frac{d}{d t} \hat{\Omega}(t)
$$

Noting equation (17), equation (16a) leaves one a simple form

$$
\begin{gathered}
\frac{d}{d t} \hat{\Omega}(t)=\omega_{1}(t)\left\{h_{x}(t) \cos \alpha(t) \sin \beta(t)\right. \\
\left.+h_{y}(t) \sin \alpha(t) \sin \beta(t)+h_{z}(t) \cos \beta(t)\right\}
\end{gathered}
$$

The equation (18) can be integrated conveniently because all the operators in the equation belong the LOMSO subspace. By employing the initial condition

\footnotetext{
${ }^{10}$ The incorrect equations (16) appearing in the original manuscript and its published paper are

$$
\begin{gathered}
\frac{d}{d t} \cos \left(\frac{1}{2} \hat{\Omega}(t)\right)=-\omega_{1}(t) \sin \left(\frac{1}{2} \hat{\Omega}(t)\right)\left\{h_{x}(t) \cos \alpha(t) \sin \beta(t)\right. \\
\left.+h_{y}(t) \sin \alpha(t) \sin \beta(t)+h_{z}(t) \cos \beta(t)\right\} \\
\frac{d}{d t}\left[\cos \alpha(t) \sin \beta(t) \sin \left(\frac{1}{2} \hat{\Omega}(t)\right), \sin \alpha(t) \sin \beta(t) \sin \left(\frac{1}{2} \hat{\Omega}(t)\right), \cos \beta(t) \sin \left(\frac{1}{2} \hat{\Omega}(t)\right)\right] \\
=\frac{1}{2} \omega_{1}(t) \cos \left(\frac{1}{2} \hat{\Omega}(t)\right)\left[h_{x}(t), h_{y}(t), h_{z}(t)\right] \\
+\frac{1}{2} \omega_{1}(t) \sin \left(\frac{1}{2} \hat{\Omega}(t)\right)\left[h_{z}(t) \sin \alpha(t) \sin \beta(t)-h_{y}(t) \cos \beta(t),\right. \\
h_{z}(t) \cos \alpha(t) \sin \beta(t)-h_{x}(t) \cos \beta(t), \\
\left.h_{x}(t) \sin \alpha(t) \sin \beta(t)-h_{y}(t) \cos \alpha(t) \sin \beta(t)\right]
\end{gathered}
$$

${ }^{11}$ The incorrect operator variable appearing only in the published paper is $\sin \alpha(t) \sin \beta(t)$ : $\sin \left(\frac{1}{2} \hat{\Omega}(t)\right)$
} 
$\hat{\Omega}(0)=0$ the integration is written a: 12

$$
\hat{\Omega}(t)=\int_{0}^{t} d t^{\prime}\left\{\omega_{1}\left(t^{\prime}\right) \boldsymbol{h}\left(\boldsymbol{t}^{\prime}\right) \cdot \boldsymbol{g}\left(\boldsymbol{t}^{\prime}\right)\right\}
$$

where the operator vector with unit magnitude $\boldsymbol{g}(\boldsymbol{t})=(\cos \alpha(t) \sin \beta(t), \sin \alpha(t)$ $\times \sin \beta(t), \cos \beta(t))$.

Now the eigenvalues of the operator $\Omega(t)$ in the Magnus solution (9) are readily calculated in the direct product basis of eigenbase $|i\rangle$ and $|s\rangle$ of singlespin angular momentum operators $I_{k z}$ and $S_{z}$ by diagonalizing the operator $\Omega(t)$ (see Eq. (11))

$$
\lambda_{i}^{s}(t)=\left\langle i\left|\left\langle\Psi_{s}|\Omega(t)| \Psi_{s}\right\rangle\right| i\right\rangle=\left\langle i\left|\left\langle s\left|\hat{\Omega}(t) S_{z}\right| s\right\rangle\right| i\right\rangle=m_{s}\langle i|\hat{\Omega}(t)| i\rangle
$$

where the magnetic quantum number $m_{s}=+1 / 2$ and $-1 / 2$ for $s=0$ and 1 , respectively. Because of the unit magnitude of the operator vectors $\boldsymbol{h}(\boldsymbol{t})$ and $\boldsymbol{g}(\boldsymbol{t})$ it follows from Eq. (19) that each eigenvalue of the operator $\hat{\Omega}(t)$ is bounded by

$$
|\langle i|\hat{\Omega}(t)| i\rangle| \leq \int_{0}^{t} d t^{\prime}\left|\omega_{1}\left(t^{\prime}\right)\right|
$$

This inequality (21) together with equation (20) can lead to directly the desired result by the triangle inequality:

$$
\left|\lambda_{i}^{s}(t)-\lambda_{j}^{s^{\prime}}(t)\right| \leq\left|\lambda_{i}^{s}(t)\right|+\left|\lambda_{j}^{s^{\prime}}(t)\right| \leq \int_{0}^{t} d t^{\prime}\left|\omega_{1}\left(t^{\prime}\right)\right|
$$

By comparing the inequality (22) with the Magnus criterion one obtains the explicit criterion of convergence of the Magnus expansion in the interaction frame:

$$
I(t)=\int_{0}^{t} d t^{\prime}\left|\omega_{1}\left(t^{\prime}\right)\right|<2 \pi
$$

The criterion (23) is the same as the Maricq's criterion (1) and its derivation is also built on the same scheme as the Maricq's in the case of single-spin systems. However, it should be pointed out that in the Maricq's derivation both $\boldsymbol{h}(\boldsymbol{t})$ and $\boldsymbol{g}(\boldsymbol{t})$ in Eq. (19) are the vectors of simple scalars, while in the present situation both the two vectors $\boldsymbol{h}(\boldsymbol{t})$ and $\boldsymbol{g}(\boldsymbol{t})$ are the operator vectors of the LOMSO operator subspace.

When a time-dependent shaped RF pulse such as Gaussian pulse [10] and sech pulse [6] applied to the coupled spin system has a positive amplitude $\omega_{1}(t) \geq 0$ over the whole pulse duration $[0, t]$ the integration $I(t)$ equals actually flip angle of the shaped RF pulse. Then the Magnus expansion in the interaction frame converges and the Magnus solution of Eq. (9) exists when

\footnotetext{
${ }^{12}$ The incorrect equation (19) appearing only in the published paper is

$$
\hat{\Omega}(t)=\int_{0}^{t} d t^{\prime}\left\{\omega_{1}\right\}\left(t^{\prime}\right) \boldsymbol{h}\left(\boldsymbol{t}^{\prime}\right) \cdot \boldsymbol{g}\left(\boldsymbol{t}^{\prime}\right)
$$
}


flip angle of 13 such shaped pulse is smaller than $2 \pi$, that is, the criterion (23) is met. Evidently, the explicit criterion (23) serves as a general lower limit for the convergence radius of the Magnus expansion in the interaction picture [9]. Thus, that the condition (23) is not fulfilled does not necessarily imply that the Magnus expansion diverges and the Magnus solution of Eq. (9) does not exist. The Magnus solution exists really as long as the Magnus criterion is satisfied [1]. However, the Magnus expansion always converges and hence the Magnus solution, i.e., the exponential propagator $U_{I}(t)$ of Eq. (9) in the interaction picture exists certainly when the criterion (23) is met. Therefore, the exponential propagator of Eq. (9) and the decomposed propagator of Eq. (11) can be used to determine exactly time evolution of the coupled spin system $\mathrm{S}_{n} \mathrm{AMX}$... under a non-negative-amplitude shaped RF pulse such as Gaussian [10] and sech [6] pulses applied to the spin $\mathrm{S}$ when flip angle of the shaped RF pulse is smaller than $2 \pi$, a very popular situation in high-resolution nuclear magnetic resonance spectroscopy.

For a weak-amplitude time-dependent RF field the dot product of the two operator vectors $\boldsymbol{h}(\boldsymbol{t})$ and $\boldsymbol{g}(\boldsymbol{t})$ in equation (19) is usually nearly equal to unit operator. As a consequence, each eigenvalue of the operator $\hat{\Omega}(t)$ is evaluated approximatively by 14

$$
\langle i|\hat{\Omega}(t)| i\rangle \approx \int_{0}^{t} d t^{\prime} \omega_{1}\left(t^{\prime}\right)
$$

Then the explicit criterion (23) is reduced to the following inequality

$$
\theta(t)=\int_{0}^{t} d t^{\prime} \omega_{1}\left(t^{\prime}\right)<2 \pi
$$

\footnotetext{
${ }^{13}$ Here drops a redundant original word.

${ }^{14}$ The approximate formula $(24)$ is not easy to understand. When the amplitude $\omega_{1}(t)$ of an RF field is small, the norm $\left\|H_{i}(t)\right\|$ that is proportional to $\left|\omega_{1}(t)\right|$ is also small, as can be seen in (8). Then in that case the Magnus expansion is quickly convergent [8] and thus one may use reasonably the lowest-order approximation formula to calculate the operator $\Omega(t)$,

$$
\Omega(t) \approx \int_{0}^{t} d t^{\prime} H_{i}\left(t^{\prime}\right)
$$

Now the operator $\Omega(t)$ may be diagonalized by using the unitary transformation obtained from Eqs. (9) and (11). On the other hand, the interaction Hamiltonian $H_{i}(t)$ also can be diagonalized by using the unitary transformation obtained from Eqs. (2) and (6). One therefore obtains from the lowest-order approximation formula that

$$
\hat{\Omega}(t) S_{z} \approx \int_{0}^{t} d t^{\prime}\left\{\omega_{1}\left(t^{\prime}\right) V\left(t, t^{\prime}\right)^{+} S_{z} V\left(t, t^{\prime}\right)\right\} .
$$

Here $V\left(t, t^{\prime}\right)$ is a unitary operator. In the trivial case that the operator $V\left(t, t^{\prime}\right)$ is the unit operator the formula (24) holds naturally. In a general case the unitary operator $V\left(t, t^{\prime}\right)$ may be written as

$$
V\left(t, t^{\prime}\right)=\exp \left\{-i\left(V_{x}^{I}\left(t, t^{\prime}\right) S_{x}+V_{y}^{I}\left(t, t^{\prime}\right) S_{y}+V_{z}^{I}\left(t, t^{\prime}\right) S_{z}\right)\right\}
$$

Here $V_{p}^{I}\left(t, t^{\prime}\right)(p=x, y, z)$ are the LOMSO operators of the subsystem AMX... Note that the amplitude function $\omega_{1}(t)$ and phase function $\phi(t)$ in (2) can be taken as arbitrary functions, respectively. Then the lowest-order approximation formula shows that one must set $V_{x}^{I}\left(t, t^{\prime}\right) \approx$ 0 and $V_{y}^{I}\left(t, t^{\prime}\right) \approx 0$. This directly leads the lowest-order approximation formula to the formula
} (24). 
This criterion shows that the Magnus expansion converges and the Magnus solution (9) exists when flip angle $\theta(t)$ of a weak-amplitude time-dependent RF pulse is smaller than $2 \pi$, that is, the inequality (25) is met. The criterion (25) may be useful for the case of those shaped RF pulses such as sinc and hermite pulses [4] whose amplitude $\omega_{1}(t)$ may not be always positive over the whole pulse duration.

The real unknown parameters in the propagators of Eqs. (9), (11), and (15) can be generally determined by solving the linear differential equations (16). It should be noted that the linear differential equations (16) are not independent but in which the four operator variables are constrained 15 by

$$
\begin{gathered}
{\left[\cos \left(\frac{1}{2} \hat{\Omega}(t)\right)\right]^{2}+\left[\cos \alpha(t) \sin \beta(t) \sin \left(\frac{1}{2} \hat{\Omega}(t)\right)\right]^{2}} \\
+\left[\sin \alpha(t) \sin \beta(t) \sin \left(\frac{1}{2} \hat{\Omega}(t)\right)\right]^{2}+\left[\cos \beta(t) \sin \left(\frac{1}{2} \hat{\Omega}(t)\right)\right]^{2}=E
\end{gathered}
$$

where $E$ is unit operator.

Actually, the propagator $U_{I}(t)$ of Eq. (5) can be generally expressed as a sum of the base operators of the operator subspace $G$

$$
U_{I}(t)=\hat{f}(t)-2 i \sum_{p} \hat{g}_{p}(t) S_{p} \quad(p=x, y, z)
$$

where the operators $\hat{f}(t)$ and $\hat{g}_{p}(t)$ are the LOMSO operators. Because of the unitarity of the propagator $U_{I}(t)$ the operators are written respectively in form as $\hat{f}(t)=\cos \left(\frac{1}{2} \tilde{\Omega}(t)\right) ; \hat{g}_{x}(t)=\cos \tilde{\alpha}(t) \sin \tilde{\beta}(t) \sin \left(\frac{1}{2} \tilde{\Omega}(t)\right), \hat{g}_{y}(t)=\sin \tilde{\alpha}(t) \sin \tilde{\beta}(t)$ $\times \sin \left(\frac{1}{2} \tilde{\Omega}(t)\right)$, and $\hat{g}_{z}(t)=\cos \tilde{\beta}(t) \sin \left(\frac{1}{2} \tilde{\Omega}(t)\right)$, where $\tilde{\alpha}(t), \tilde{\beta}(t)$, and $\tilde{\Omega}(t)$ are still the LOMSO operators. Obviously, $\tilde{\alpha}(t)=\alpha(t), \tilde{\beta}(t)=\beta(t)$, and $\tilde{\Omega}(t)=$ $\hat{\Omega}(t)$ when the Magnus solution (9) exists. By inserting the expansion-form propagator of Eq. (26) into the Schrödinger equation (14) one can still obtain the same linear differential equations as equations (16) but with the new operator variables $\hat{f}(t)$ and $\hat{g}_{p}(t)(p=x, y, z)$. The differential equations are entirely equivalent to the Schrödinger equation (14) and can determine an alternative solution for the Magnus solution (9) to the time-dependent Schrödinger equation [9]. Because the solution to the differential equations always exists one can obtain the unknown parameters in the propagator of Eq. (26) by solving the differential equations. Thus, the expansion-form propagator of Eq. (26) always exists and can be generally used to determine time evolution of the coupled spin system under an arbitrary time-dependent RF pulse no matter whether the criterion (23) is met or not. This indicates that the time evolution can be described in the small operator algebra subspace $G=u(2) \bigotimes L O M S O$ instead of 16 the whole Liouville operator space of the system. Thus, this simplifies greatly determination of the time evolution.

\footnotetext{
${ }^{15}$ The original manuscript's word is corrected here.

${ }^{16}$ Here drops a redundant original word.
} 
In general, the integration $I(t)$ is always more than flip angle $\theta(t)$ for a shaped pulse. Then before employing the criterion (23) one usually needs to first calculate explicitly the integration $I(t)$ for complex shaped selective pulses such as the BURP pulses [11] and the Gaussian pulse cascades [12, 13] whose amplitude functions $\omega_{1}(t)$ are not always non-negative over the whole pulse duration even if their flip angles given in advance are smaller than $2 \pi$. The numerical calculation 17 shows that the criterion (23) is met for the excitation pulses $G^{4}$ and $Q^{5}$ of the Gaussian pulse cascades $[12,13]$ but is violated for the BURP pulses [11] including E-BURP, U-BURP, I-BURP, RE-BURP pulses and the Gaussian pulse cascades $G^{3}$ and $Q^{3}[12,13]$. It should be pointed out that the violation of the criterion (23) for these pure-phase shaped selective pulses does not imply that the exponential propagator of Eq. (9) can not be used to describe time evolution of the coupled spin system under these shaped pulses. However, as an alternative one still can use the expansion-form propagator of Eq. (26) of the smaller operator subspace $G=u(2) \bigotimes L O M S O$ instead of that one of the whole Liouville operator space of the coupled spin system $\mathrm{S}_{n}$ AMX... to determine generally time evolution of the spin system under those shaped pulses and shaped decoupling sequences [17] that do not satisfy the criterion (23).

\section{Acknowledgement}

This work was supported by the NSFC general projects with grant number 19974064. Author thanks the referees for their valuable suggestions.

\section{References}

[1] W. Magnus, Commun. Pure Appl. Math. 7 (1954) 649

[2] R. R. Ernst, G. Bodenhausen, and A. Wokaun, Principles of nuclear magnetic resonance in one and two dimensions (Oxford University Press, Oxford, 1987)

[3] U. Haeberlen, High resolution NMR in solids, Adv. Magn. Reson. Suppl. 1 (1976)

[4] W. S. Warren, J. Chem. Phys. 81 (1984) 5437

[5] X. Miao and R. Freeman, J. Magn. Reson. A 119 (1996) 90

[6] X. Miao, J. Chen, and X. A. Mao, Chem. Phys. Lett. 304 (1999) 45

[7] M. M. Maricq, Adv. Magn. Reson. 14 (1990) 151

[8] M. M. Maricq, Phys. Rev. B 25 (1982) 6622

[9] M. M. Maricq, J. Chem. Phys. 86 (1987) 5647

[10] C. Bauer, R. Freeman, T. Frenkiel, J. Keeler, and A. J. Shaka, J. Magn. Reson. 58 (1984) 442

[11] H. Geen and R. Freeman, J. Magn. Reson. 93 (1991) 93

[12] L. Emsley and G. Bodenhausen, Chem. Phys. Lett. 165 (1990) 469

\footnotetext{
${ }^{17}$ The numerical calculation merely illustrates that some shaped pulses may satisfy the criterion (23), while some others also may violate that criterion in the numerical calculation condition.
} 
[13] L. Emsley and G. Bodenhausen, J. Magn. Reson. 97 (1992) 135

[14] X. Miao, Molec. Phys. (in press)

[15] X. Miao, X. Han, and J. Hu, Sci. China A 36 (1993) 1199

[16] X. Miao and C. Ye, Molec. Phys. 90 (1997) 499

[17] R. Freeman, Spin Choreography (Spektrum, Oxford, 1997) 